\title{
Un code postal pour les enzymes lysosomales
}

Les hydrolases acides ont un cheminement complexe entre leur synthèse sur le réticulum endoplasmique rugueux et leur transport, après glycosylation et démasquage de résidus mannose-6-phosphate, vers le lysosome. Ce processus est anormal dans une maladie, la mucolipidose II, caractérisée par l'absence de mannose-6-phosphate libre sur les hydrolases acides, qui sont alors sécrétées dans le milieu extracellulaire et non plus concentrées dans les lysosomes.

\section{Philippe Crine \\ Professeur agrégé}

\section{Lucie Des Parois Stagiaire de recherche}

\section{Hélène Lecavalier Stagiaire de recherche}

\author{
ADRESSE \\ P. Crine, L. Des Parois, H. Lecavalier : \\ département de biochimie et groupe de recher- \\ che en transport membranaire, université de \\ Montréal C.P. 6128 succ. A, Montréal QC, \\ Canada H3C 3J7.

es lysosomes sont des organites cytoplasmiques que l'on retrouve dans toutes les cellules eucaryotes où ils sont impliqués dans la dégradation de nombreux consţituants macromoléculaires [1]. Étudiés au microscope électronique, ils ont l'aspect de vésicules limitées par une membrane simple, de forme et de taille très variables [2]. Ces vésicules renferment un ensemble d'enzymes hydrolytiques capables de digérer toutes les classes de macromolécules. On connaît en fait une cinquantaine d'hydrolases lysosomales différentes, incluant des nucléases, des glycosidases, des protéases, des lipases, des phospholipases, des phosphatases et des sulfatases. Toutes ces enzymes ont une activité optimale à $\mathrm{pH}$ voisin de 5,0 , qui est le $\mathrm{pH}$ existant à l'intérieur du lysosome. On parlera donc d'hydrolases acides.

L'hétérogénéité morphologique des lysosomes reflète la diversité des fonctions que remplissent les hydrolases acides dans la cellule. Chaque type de lysosome est caractérisé par un ensemble d'hydrolases acicies qui lui est propre. Certains lysosomes peuvent ainsi être impliqués dans la dégradation et le renouvellement de constituants intra- et extracellulaires. D'autres jouent un rôle dans la digestion des microorganismes après leur phagocytose par la cellule. D'autres encore sont responsables de l'autolyse de certaines cellules dans le programme de développement embryogénétique. Les lysosomes peuvent également être chargés de l'assimilation de cholestérol après l'internalisation des lipoprotéines sériques.

Les premières études sur la biogenèse des lysosomes ont montré que ces organites se formaient à partir des vésicules qui bourgeonnaient de l'appareil de Golgi ou d'une structure tubulaire à membrane lisse associée à l'appareil de 


\section{RÉFÉRENCES}

1. De Duve C. Exploring cells with a centrifuge. Science $1975 ; 189$ : 186-94.

2. Novikoff AB, Novikoff PM. Cytochemical contributions to differentiating GERL from the Golgi apparatus. Histochem $J 1977$; 7 : 525-51.

3. Walter P, Gilmore R, Blobel G. Protein translocation across the endoplasmic reticulum Cell $1984 ; 38$ : 5-8.

4. Neufeld EF, Lim TW, Shapiro LJ. Inherited disorders of lysosomal metabolism. Ann Rev Biochem 1975 ; 44 : 357-75.

5. Hickman S, Neufeld EF. An hypothesis for I-cell disease : defective hydrolases that do not enter lysosomes. Biochem Biophys Res Commun 1972 ; 49 : 992-9.

6. Hickman S, Shapiro LJ, Neuf eld EF. A recognition marker for uptake of a lysosomal enzyme by cultured fibroblasts. Biochem Biophys Res Commun 1974 ; 57 : 55-61.

7. Kaplan A, Achord DT, Sly W. Phospho hexosyl components of a lysosomal enzyme are recognized by pinocytosis receptors on human fibroblasts. Proc Nall Acad Sci USA $1977 ; 74$ : 2026-30

8. Varki A, Kornfeld S. Structural studies of phosphorylated high mannose-type oligosaccharides. J Biol Chem 1980 ; 255 : 10847-58.

9. Fisher HD, Gonzalez-Noriega A, Sly WS Morré DJ. Phosphomannosyl-enzyme receptors in rat liver : subcellular distribution and role in intracellular transport of lysosomal enzymes. $J$ Biol Chem 1980 ; 255 : 9608-15.

10. Tabas I, Kornfeld S. Biosynthetic intermediates of $\beta$-glucuronidase contain high mannose oligosaccharides with blocked phosphate residues. J Biol Chem 1980 ; 255 : 6633-9.

11. Lang L, Reitman M, Tang J, Roberts RM, Kornfeld S. Lysosomal enzyme phosphorylation : recognition of a protein dependent determinant allows specific phosphorylation of oligosaccharides present on lysosomal enzymes. J Biol Chem 1984; 259 : 14663-71.

12. Minnifield N, Creek KE, Navas $P$, Morré $\mathrm{DJ}$. Involvement of cis and trans Golgi apparatus elements in the intracellular sorting and targeting of acid hydrolases to lysosomes. Europ J Cell Biol 1986 ; 42 : 92-100.

13. Sahagian GC, Distler JJ, Jourdian GW. Characterization of a membrane-associated receptor from bovine liver that binds phosphomannosyl residues of bovine testicular $\beta$ galactosidase. Proc Natl Acad Sci USA 1981 ; 78 : 4289-93.

14. Geuze HJ, Slot JW, Strous GJAM, Hasilik A, von Figura K. Ultrastructural localization of the mannose-6-phosphate receptor in rat

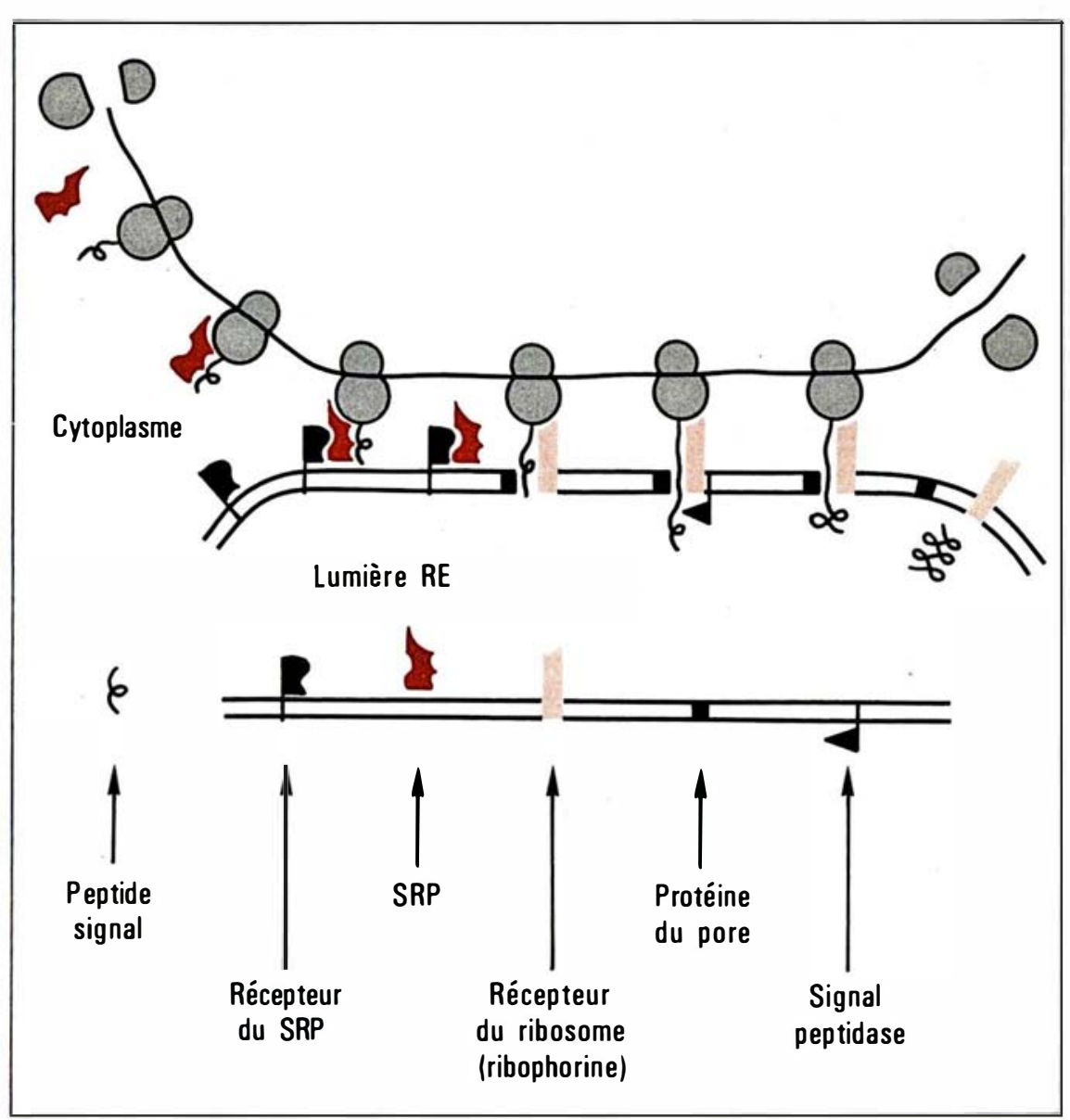

Figure 1. Translocation des hydrolases acides dans la lumière du réticuIum endoplasmique (RE) rugueux. L'initiation de la synthèse protéique débute dans le cytoplasme. Dès que l'extrémité $\mathrm{N}$-terminale de la protéine émerge de la grosse sous-unité du ribosome, une particule cytosolique (SRP : signal recognition particule) $s^{\prime} y$ attache et bloque l'élongation de la chaîne polypeptidique. La synthèse protéique reprend lorsque l'ensemble s'associe à un récepteur de la membrane du réticulum reconnaissant le SRP $\left(\mathrm{m} / \mathrm{s}, n^{\circ} 6\right.$, vol. 2, p. 341). La protéine naissante est alors insérée à travers un pore protéique de la membrane et poussée dans la lumière du réticulum endoplasmique. L'association du ribosome à la membrane pourrait être éventuellement prise en charge par des ribophorines du réticulum endoplasmique rugueux. L'extrémité $\mathrm{N}$-terminale de la protéine appelée peptide signal est très vite éliminée de la protéine en voie d'élongation grâce à une peptidase située sur la face interne de la membrane du réticulum.

Golgi et au réticulum endoplasmique (GERL). Plus récemment, plusieurs équipes se sont attachées à élucider la manière dont les hydrolases acides étaient synthétisées puis concentrées à l'intérieur des lysosomes. Les mécanismes moléculaires se trouvant à la base de ces phénomènes sont à présent compris de façon assez détaillée et nous voulons en faire ici une brève revue.

Les hydrolases acides, comme tou- tes les autres protéines destinées à être excrétées de la cellule ou insérées dans la membrane plasmique, sont synthétisées sur les ribosomes liés au réticulum endoplasmique. Les protéines de ce groupe disposent généralement d'une extension de 20 acides aminés, le plus souvent située à leur extrémité aminée. Cette séquence d'acides aminés, appelée " peptide signal ", permet au polypeptide en voie d'élongation de s'ancrer à 
des récepteurs situés dans la membrane du réticulum endoplasmique (figure 1). Ces protéines sont ensuite transférées dans la lumière de cet organite où l'extension $\mathrm{N}$-terminale est, dans la plupart des cas, éliminée [3]. Parmi toutes les protéines synthétisées sur les ribosomes liés aux membranes et insérées dans la lumière du réticulum endoplasmique, la cellule doit alors reconnaître spécifiquement les hydrolases acides pour les trier du reste des protéines et les orienter de façon appropriée vers les lysosomes.

Par analogie avec le "peptide signal ", on peut imaginer que tous ces phénomènes pourraient être contrôlés par la présence d'éléments d'information ou " signaux " intégrés à la structure même des protéines. Ces signaux seraient reconnus par des systèmes récepteurs "qui assurent la ségrégation des protéines pour permettre leur voyage vers le compartiment cellulaire qui constitue leur destination finale. Ces signaux joueraient donc le rôle d'un véritable " code postal " destiné à diriger le transport intracellulaire des protéines. La structure chimique du " code postal " des hydrolases acides est à présent connue, et le récepteur chargé de sa lecture a été identifié. Ces découvertes constituent donc des précédents importants pour les travaux sur les mécanismes assurant le tri des autres protéines.

\section{Découverte des résidus mannose-6-phosphate}

L'existence d'un marqueur qui serait propre aux hydrolases acides et permettrait leur reconnaissance par un mécanisme de tri a pu être mise en évidence grâce à l'étude de diverses maladies affectant les fonctions lysosomales. Ainsi, lorsqu'on analyse le contenu lysosomal des cellules de patients atteints de mucopolysaccharidose, on constate l'absence d'une enzyme responsable du catabolisme des mucopolysaccharides. A partir de biopsies de la peau, on peut maintenir en culture des fibroblastes dont le con- tenu en hydrolases acides reflète le défaut enzymatique caractéristique de la maladie. Dès la fin des années 60 , on savait qu'il était possible de compenser la carence en $\beta$-glucuronidase des fibroblastes de patients atteints de mucopolysaccharidose en ajoutant l'enzyme provenant d'individus normaux au milieu de culture où ces cellules étaient maintenues [4]. Ce processus de capture, de par sa sélectivité et sa saturabilité, suggérait alors que la reconnaissance de ces enzymes dépendait d'un récepteur situé à la surface cellulaire

D'autre part, chez certains patients souffrant d'une maladie héréditaire récessive, la mucolipidose II, on avait constaté une déficience intracellulaire en de multiples activités hydrolytiques. Les fibroblastes de ces patients étaient pourtant capables de synthétiser ces enzymes qui étaient actives mais étaient sécrétées dans le milieu extra-cellulaire plutôt que d'être dirigées vers le lysosome. Cette déficience multiple en diverses hydrolases acides pouvait également être corrigée en fournissant aux fibroblastes malades cultivés in vitro les enzymes de sujets normaux. On ne pouvait donc expliquer cette déficience par l'absence ou l'inactivité du récepteur responsable de la recapture. Cette maladie, qui a un caractère récessif, résulte probablement d'une mutation au niveau d'un gène unique. Cela suggérait donc que la mucolipidose II était due à un défaut au niveau du signal de reconnaissance qui devrait donc être commun à plusieurs, sinon à toutes les hydrolases acides [5].

Le phenomène de recapture des hydrolases acides exogènes peut être inhibé par la destruction préalable de leur composante oligosaccharidique sous l'action du periodate [6]. Cela laissait supposer que le " marqueur " reconnu par les récepteurs à la surface des fibroblastes en culture était situé dans la partie oligosaccharidique de cette classe particulière de glycoprotéines que constituent les hydrolases acides. En se basant sur ce premier indice, Kaplan et ses collaborateurs [7] ont alors pu démontrer que la liaison des hydrolases acides exogènes à leurs récepteurs sur les fibroblastes pouvait être spécifiquement inhibée par l'addition de mannose-6-phosphate (man-6-P) dans le milieu de culture. L'addition d'autres sucres tels le mannose, le glucose-6-phosphate ou le galactose n'avait aucun effet sur le phénomène. Ces résultats constituaient la première indication que le marqueur propre aux hydrolases acides était constitué de résidus man-6-P. Ces groupements particuliers ont plus tard été identifiés chimiquement au sein de la structure des oligosaccharides, liés aux hydrolases acides [8] mais ils n'ont jamais été retrouvés dans aucun autre type de glycoprotéines.

\section{La voie d'insertion dans le lysosome}

L'existence de résidus mannose-6phosphate (man-6-P) dans les hydrolases acides permet donc leur liaison spécifique aux récepteurs situés à la surface des fibroblastes et leur internalisation sélective dans des structures vésiculaires qui, pour finir, fusionneraient avec les lysosomes à l'intérieur de la cellule. A partir de cette observation, on pourrait donc imaginer un processus selon lequel les enzymes lysosomales seraient d'abord sécrétées avant d'être recapturées par les cellules (hypothèse de la sécrétion-recapture).

Cependant, si on ajoute au milieu de culture des fibroblastes normaux un excès de man-6-P libre capable d'inhiber la recapture d'hydrolases acides qui pourraient éventuellement avoir été sécrétées dans le milieu de culture, on n'affecte pas le contenu enzymatique des lysosomes. Il semble donc que les hydrolases acides nouvellement synthétisées soient insérées selon une voie exclusivement intracellulaire sans qu'il y ait sécrétion préalable des enzymes à l'extérieur des cellules. L'élaboration d'un test de liaison des hydrolases acides à leur récepteur a permis de confirmer cette 


\section{RÉFÉRENCES}

15. Brown WJ, Farquhar MG. The mannose-6-phosphate receptor for lysosomal enzymes is concentrated in cis Golgi cisternae. Cell $1984 ; 86$ : 295-307.

16. Holfack B, Kornfeld S. Purification and characterization of a cation-dependant mannose-6-phosphate receptor from murine P388D, macrophages and bovine liver. $J$ Biol Chem 1985 ; 260 : 12008-14

17. Holfack B, Fujimoto K. Kornfeld S. The interaction of phosphorylated oligosaccharides and lysosomal enzymes with bovine liver cation-dependent mannose-6-phosphate receptor. J Biol Chem 1987 ; 262 : 123-9.

18. Brown MD, Anderson RGW. Goldstein JL. Recycling receptors : the round trip itinerary of migrant membrane proteins. Cell 1983 ; 32 : 663-7.

19. Brown WJ, Farquhar MG. Accumulation of coated vesicles bearing mannose-6-phosphate receptors for lysosomal enzymes in the Golgi region of I cell fibroblats. Proc Nall Acad Sa USA 1984; 81 : 5135-9.

20. Brown WJ, Goodhouse J, Farquhar MG Mannose-6-phosphate receptors for lysosomal enzymes cycle between the Golgi complex and endosomes. J Cell Biol 1986 ; 103 : 1235-47.

21. Sly WS, Fisher HD. The phosphomannosyl recognition system for intracellular and intercellular transport of lysosomal enzymes. $J$ Cell Biochem 1982 ; 18 : 67-85.

22. Hasilik A, Neufeld EF. Biosynthesis of lysosomal enzymes in fibroblasts. Synthesis as precursors of higher molecular weight. $J$ Biol Chem 1980 ; 255 : 4937-45.

23. Grieselman V, Hasilik A, von Figura $\mathrm{K}$ Processing of human cathepsin D in lysosomes in vitro. $J$ Biol Chem 1985 ; 260 : 3215-20.

24. Gabel CA, Foster SA. Lysosomal enzyme trafficking in mannose-6-phosphate receptorpositive mouse L-cells : démonstration of a steady state accumulation of phosphorylated acid hydrolases. J Cell Biol 1986 ; 102 : 943-50.

25. Gabel CA, Foster SA. Mannose-6-phosphate receptor-mediated endocytosis of acid hydrolases : internalization of $\beta$-glucuronidase is accompanied by a limited dephosphorylation. J Cell Biol 1986; 103 : 1817-27.

26. Lemansky P, Grieselman V, Hasilik A von Figura K. Synthesis and transport of lysosomal acid phosphatase in normal and I-cell fibroblats. J Biol Chem 1985; 260 : 9023-30 hypothèse en démontrant que le nombre de récepteurs présents à la surface cellulaire ne représente que $10 \%$ de celui mesuré sur les fractions cellulaires correspondant aux membranes intracellulaires (réticulum endoplasmique rugueux, Golgi, membranes lysosomales). De plus, il semble que la proportion de récepteurs engagés dans une liaison avec une hydrolase acide va en décroissant dans l'ordre suivant : réticulum endoplasmique, Golgi, lysosome [9]. Les récepteurs présents dans les lysosomes et ceux situés dans la membrane plasmique ne présentent qu'un taux d'occupation de $10 \%$ alors que ce taux d'occupation est de $80 \%$ dans le réticulum. Ces résultats sont donc compatibles avec un modèle selon lequel les enzymes lysosomales seraient transportées du réticulum endoplasmique rugueux aux lysosomes en passant par l'appareil de Golgi. Le complexe récepteurenzyme se dissocierait ensuite pour libérer les hydrolases acides dans le lysosome. L'appareil de Golgi jouerait donc un rôle essentiel au niveau du tri moléculaire responsable de l'insertion des enzymes lysosomales.

\section{Biogenèse du marqueur mannose-6-phosphate}

Lors de leur passage dans le réticulum endoplasmique, les enzymes lysosomales nouvellement synthétisées sont glycosylées au niveau de leurs résidus asparagine. Comme pour toutes les autres glycoprotéines, cette addition d'oligosaccharides s'opère par le transfert en bloc d'un oligosaccharide riche en mannose. Les glycoprotéines sont ensuite acheminées vers l'appareil de Golgi, où elles subissent certaines modifications additionnelles au niveau de leurs chaînes oligosaccharidiques. Dans le cas des hydrolases acides, l'une de ces modifications consiste en l'addition du marqueur man-6-P. Cette addition procède en deux étapes [10]. La première consiste à transférer un résidu N-acétylglucosamine-1phosphate (GlcNac-1-P) à partir de l'uridine-diphosphate-N-acétylglucosamine (UDP-N-acétylglucosamine) vers la position 6 de certains résidus mannose dans l'oligosaccharide initial. Dans la deuxième étape, il y a hydrolyse du pont phosphoester entre le groupement phosphate et le GlcNac, ce qui découvre ainsi le résidu man-6-P (figure 2). La deuxième étape, qui est catalysée par une phosphodiestérase, est essentielle pour que l'hydrolase acide se lie au récepteur du man-6-P. Si le groupement reste " coiffé " par un résidu Glc-Nac, il ne pourra être reconnu par le récepteur. L'enzyme responsable de la première étape est une GlcNac-1-phosphotransférase. Cette enzyme, tout comme la phosphodiestérase, a été localisée au niveau des premiers saccules de l'appareil de Golgi (région " cis " : là où fusionnent les vésicules issues du réticulum endoplasmique). Cette enzyme joue également un rôle clef dans le tri des hydrolases acides puisque, parmi toutes les glycoprotéines qui passent par le Golgi, elle est capable de reconnaître uniquement celles qui correspondent aux précurseurs des hydrolases acides. Une étude récente portant sur la cathepsine $\mathrm{D}$ et l'utéroferrine a démontré que la Glc-Nac-1-phosphotransférase était capable de reconnaître certains déterminants structuraux de la chaîne polypeptidique de ces protéines en l'absence des groupements oligosaccharidiques [11]. Il est probable que ces déterminants structuraux sont de nature conformationnelle. La deuxième réaction est catalysée par la $\mathrm{N}$-acétylglucosamine-1-phosphodiester $\alpha$-N-acétylglucosaminidase qui a aussi été partiellement purifiée à partir de foie de rat et de placenta humain. Cette enzyme se retrouve dans les saccules " cis " et "trans" de l'appareil de Golgi [12].

\section{Le récepteur du mannose-6-phosphate}

Le récepteur du man-6-P a pu être purifié par chromatographie d'affinité sur une colonne constituée d'hydrolases acides immobi- 


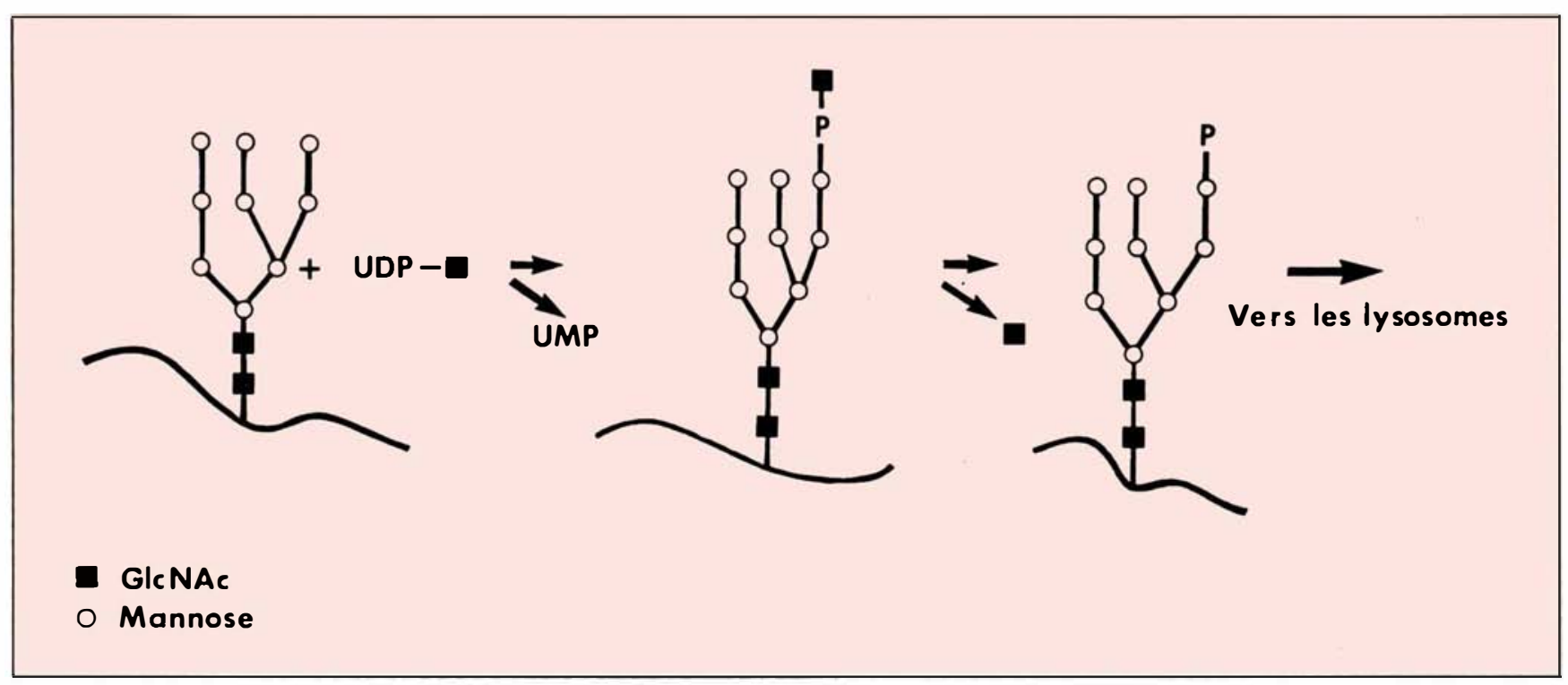

Figure 2. Phosphorylation des résidus mannose des hydrolases acides. UDP, UMP = uridine di- et mono-phosphate ; $P=$ phosphate. La première étape montrée dans cette figure transfère un résidu $\mathrm{N}$-acétylglucosamine-1-phosphate (GIcNac-1-P) à partir de l'UDP-N-acétylglucosamine vers la position 6 de certains résidus mannosé dans l'oligosaccharide présent initialement sur l'asparagine de l'hydrolase acide. Bien qu'on ait illustré ici la phosphorylation d'un seul résidu mannose, il est certain que ce phénomène se passe simultanément sur plusieurs résidus mannose d'un même oligosaccharide. La deuxième étape montre l'hydrolyse de la liaison phosphodiester entre le groupement phosphate et le résidu de $\mathrm{N}$-acétylglucosamine, démasquant le groupement man-6-P.

lisées sur un support solide [13]. Il s'agit d'une glycoprotéine membranaire dont le poids moléculaire a été évalué à 215000 . Dans les cellules parenchymateuses de foie de rat, les méthodes immunohistochimiques permettent de démontrer l'existence de ce récepteur dans toutes les membranes de l'appareil de Golgi [14].

D'autre part, on a pu montrer aussi que la cathepsine $D$ se retrouvait associée au récepteur man-6-P tout le long des saccules du Golgi. Ces observations suggèrent donc que les enzymes lysosomales associées au récepteur man-6-P traverseraient toutes les saccules de l'appareil du Golgi, de la région "cis" à la région " trans ". Cette hypothèse est également en accord avec le fait que certaines enzymes lysosomales (surtout celles sécrétées par les cellules en culture) contiennent des sucres complexes, qui sont ajoutés uniquement dans les saccules "trans" du Golgi.

Un tout autre modèle de transport $\mathrm{m} / \mathrm{s} n^{\circ} 8$ vol. 3 , octobre 87 des enzymes lysosomales a été suggéré par Brown et Farquhar [15]. Les études immunocytochimiques que les auteurs ont faites sur des cellules de pancréas exocrine et sur des hépatocytes n'ont pu mettre en évidence les récepteurs au man-6-P que dans un ou deux saccule(s), à l'extrémité " cis " de l'appareil de Golgi. Dans ce modèle, l'association des enzymes lysosomales, nouvellement synthétisées, avec les récepteurs de man-6-P dans les saccules "cis " du Golgi permettrait d'exclure les enzymes lysosomales du reste des glycoprotéines de sécrétion dès leur entrée dans cet organite. Les enzymes lysosomales, retenues par les récepteurs de man-6-P, seraient ensuite expédiées vers les lysosomes, à partir de la région "cis" sans avoir à traverser le Golgi en entier. Ce modèle est en accord avec le fait que certaines hydrolases acides sont dépourvues de sucres terminaux et ne semblent pas transiter par les saccules distaux de l'appa- reil de Golgi. Il semble donc qu'il existe des variations dans le chemin exact suivi par les hydrolases acides au cours de leur transport cellulaire et que le mécanisme précis puisse dépendre du type cellulaire où il est étudié. L'intégration de toutes les observations en un modèle unique est encore compliquée par la découverte récente d'un deuxième récepteur au man-6-P. Ce récepteur differe du premier par son poids moléculaire (46 000 au lieu de 215000 ) et le fait qu'il requiert des cations divalents pour lier le man-6-P [16, 17]. On ignore encore tout de sa distribution intracellulaire et de son rôle dans le contrôle du transport des hydrolases acides.

\section{Livraison des hydrolases acides aux lysosomes}

On commence également à connaître en détail les modalités du trajet intracellulaire suivi par les enzymes lysosomales nouvellement 
synthétisées. L'immunocytochimie démontre la présence de récepteurs au man-6-P dans des vésicules mantelées tapissées de clathrine au voisinage de l'appareil de Golgi, ainsi que dans des endosomes et des lysosomes [15]. On suppose donc que les hydro- lases acides, après s'être fixées à leur récepteur dans l'appareil de Golgi, se concentrent dans des régions particulières de la membrane de cet appareil qui sont tapissées de clathrine. Ces régions bourgeonnent et forment des vésicules mantelées contenant les enzymes lysosomales liées à leur récepteur (figure 3). Ces vésicules, qui constituent la navette de transport des hydrolases acides nouvellement synthétisées vers le lysosome, seraient donc analogues à celles qui assurent l'internalisation des ligands aux divers récep-

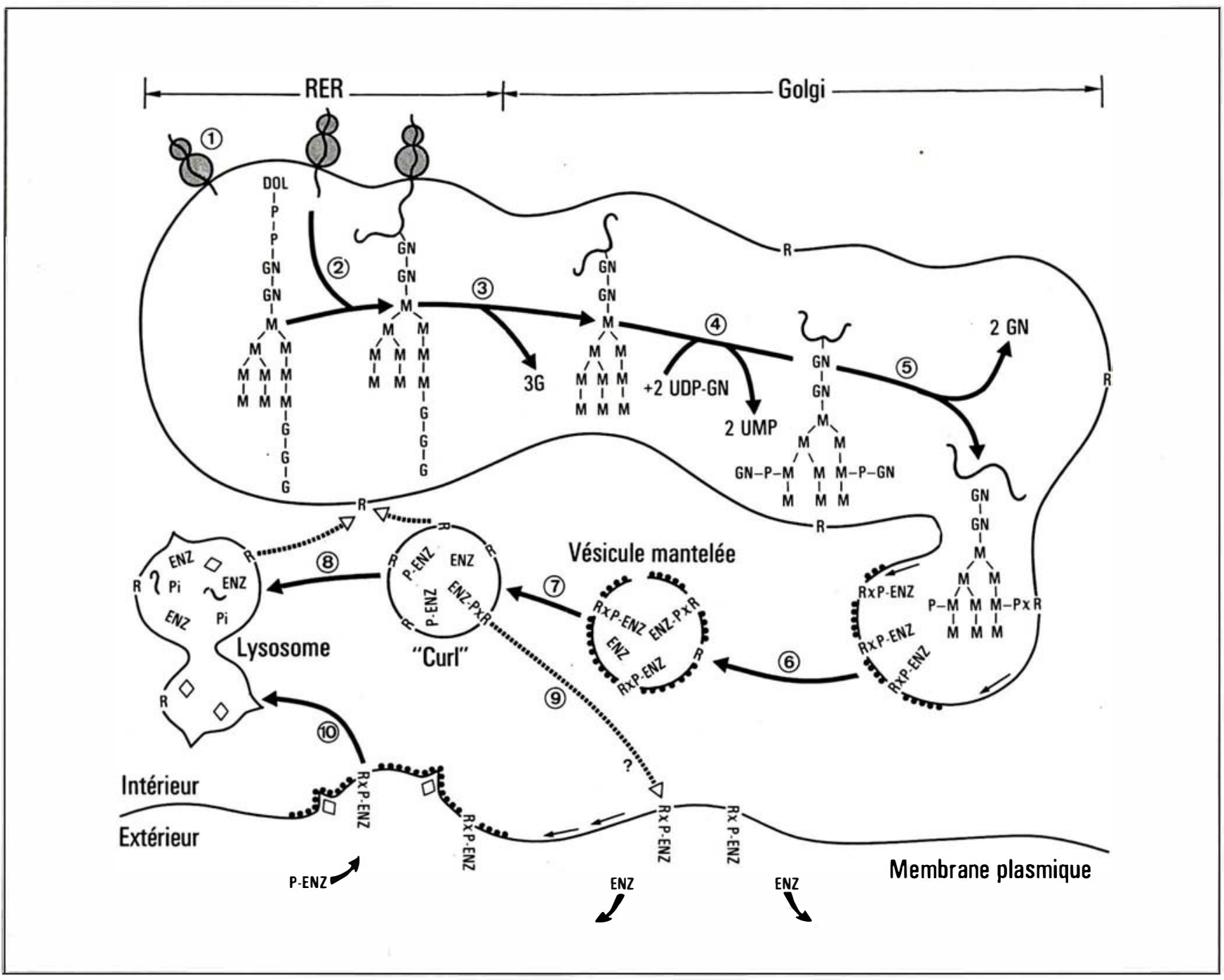

Figure 3. Mécanisme moléculaire de la biosynthèse des hydrolases acides. Les hydrolases acides synthétisées par les ribosomes liés aux membranes du réticulum endoplasmique rugueux (RER) (1) sont glycosylées par le transfert en bloc d'un oligosaccharide préformé sur un intermédiaire lipidique, le dolichol (2). Après la perte de certains résidus de sucre (3), l'oligosaccharide à haut contenu en mannose est transféré dans l'appareil de Golgi et phosphorylé selon le mécanisme de la figure 2 (4, 5). Les molécules d'enzymes ainsi marquées reconnaissent sur la face interne de la membrane de l'appareil de Golgi un récepteur qui les dirige vers des régions tapissées de clathrine. Ces vésicules bourgeonnent (6) et deviennent des vésicules mantelées. La couche de clathrine disparaît et le contenu des vésicules s'acidifie au niveau du "Curl" (compartment for uncoupling receptor and ligand) (7). Cela provoque la dissociation de l'enzyme du récepteur. Ce dernier est recyclé vers le système RER-Golgi alors que l'enzyme est livrée au lysosome (8). II est possible qu'un petit nombre de molécules d'enzymes lysosomales transitent par la surface cellulaire (9) avant d'être réinternalisées vers le lysosome (10). Cette dernière voie est particulièrement bien mise en évidence lors de l'addition d'enzymes au milieu de culture de fibroblastes in vitro. $M=$ mannose; $G=g l u c o s e ; G N=N$ acétylglucosamine ; $P=$ phosphate $; E N Z=$ enzyme $; R=$ récepteur des résidus phosphomannosyl; $D O L=$ dolichol. 
teurs de la membrane plasmique [18].

La liaison des hydrolases acides à leur récepteur dans l'appareil de Golgi et dans les vésicules mantelées semble être un événement déterminant permettant à ces dernières d'être efficacement dirigées vers les lysosomes. Dans les fibroblastes des patients atteints de mucolipidose II, les hydrolases acides qui sont synthétisées sans leur marqueur man-6-P ne peuvent se lier au récepteur et on observe que les vésicules mantelées s'accumulent au voisinage de la région du Golgi où elles prennent naissance [19]. Il semble donc que le voyage des vésicules mantelées vers leur destination finale soit déclenché par la liaison du ligand avec le récepteur. On pourrait imaginer que l'établissement de cette liaison induise les modifications nécessaires dans l'organisation moléculaire de la membrane lysosomale pour provoquer la reconnaissance des vésicules par le lysosome.

Les hydrolases acides voyagent à travers la cellule en association avec leur récepteur mais se retrouvent à l'état libre dans le lysosome. La dissociation des hydrolases acides de leur récepteur semble avoir lieu avant que les vésicules mantelées ne fusionnent avec les lysosomes, dans un organite spécialisé. Cet organite a été baptisé "CURL ", un acronyme pour l'appellation anglaise : compartment for uncoupling receptor and ligand. Dans le "CURL ", l'acidification du milieu intravésiculaire provoque la rupture de la liaison récepteur-ligand qui n'est stable qu'à des $\mathrm{pH}$ supérieurs à 6,0 . Tout comme l'association du récepteur avec son ligand était responsable de l'expédition de la navette au lysosome, la dissociation du complexe provoque le retour du récepteur à l'appareil de Golgi [20]. Ces récepteurs peuvent alors se lier à une nouvelle molécule d'hydrolase et, ainsi chargés, refaire le chemin vers le lysosome. De leur côté, les hydrolases acides qui viennent d'être livrées au lysosome subissent une hydrolyse de leur groupement man-6-P [21], ce qui empêche une nouvelle liaison des enzymes au récepteur. Une dernière étape assurant la maturation finale des enzymes lysosomales consiste en leur protéolyse ménagée pour former les espèces moléculaires complètement actives [22]. La protéolyse des hydrolases lysosomales semble se faire en plusieurs étapes après que les molécules ont quitté l'appareil de Golgi. Les dernières étapes se passent dans le lysosome [23].

Le site exact de la déphosphorylation est encore mal connu. L'hypothèse la plus simple serait que cette réaction soit contrôlée par une phosphatase présente dans le lysosome lui-même. Il existe cependant une souche de cellules murines dont les enzymes lysosomales restent phosphorylées de façon stable [24]. Il semble donc que le fait qu'une molécule rejoigne le lysosome ne soit pas suffisant pour assurer la destruction de son marqueur man-6-P. Il est possible en fait que cette étape soit antérieure à l'arrivée des enzymes dans le lysosome et ait lieu dans un compartiment endosomal [25]. Tant que la phosphatase impliquée dans cette réaction ne sera pas caractérisée, il sera cependant difficile d'en déterminer exactement le mécanisme.

\section{Existe-t-il d'autres voies de transport?}

Bien que le marqueur man-6-P semble jouer un rôle très important pour le transport de nombreuses enzymes lysosomales, plusieurs observations suggèrent que le mécanisme décrit ci-dessus ne constitue qu'une des voies possibles. Ainsi, certaines hydrolases lysosomales telles que l' $\alpha$ glucosidase et la cathepsine $D$ se retrouvent en quantités presque normales dans les fibroblastes provenant de malades atteints de mucolipidose II [22, 26]. D'autre part, dans le foie, la rate, le rein et le cerveau de ces patients, l'activité de beaucoup d'enzymes lysosomales est normale, bien que ces tissus soient, tout comme les fibroblastes, déficients en $\mathrm{N}$-acétylglucosaminyl-1-phospho- transférase [27, 28]. Enfin, lorsqu'on traite les cellules de poumons d'embryon humain (WI-38) avec du chlorure d'ammonium, on provoque la sécrétion de l'arylsulfatase sans affecter celle de la phosphatase acide [29]. Le chlolure d'ammonium, en élevant le $\mathrm{pH}$ intralysosomal, empêche la dissociation du complexe enzyme-récepteur, le recyclage du récepteur et par conséquent son retour à l'appareil de Golgi. Les hydrolases arrivant ainsi dans un Golgi "vidé " de ses récepteurs sont sécrétées, si leur insertion dépend de la présence du récepteur (comme c'est le cas pour l'arylsulfatase).

Le fait que la phosphatase acide ne soit pas affectée par ce traitement semble indiquer que son insertion dans le lysosome est indépendante des récepteurs man-6-P. On connaît encore peu de choses sur ces autres mécanismes. L'étude de la glucocérébrosidase dans une lignée de cellules monoblastiques [30] a montré que cette enzyme portait des oligosaccharides de type complexe non phosphorylés mais fortement sialylés. L'addition d'inhibiteurs de la maturation des oligosaccharides au milieu de culture des cellules empêche l'incorporation de résidus d'acide sialique sur l'enzyme et bloque son transport vers le lysosome. Il est donc possible que, dans ce cas au moins, la sialylation soit importante pour assurer un transfert correct de l'enzyme dans le lysosome.

\section{Conclusion}

Certaines maladies sont caractérisées par une déficience en une ou plusieurs enzymes lysosomales nécessaires au métabolisme normal des cellules. Cette carence enzymatique est généralement congénitale et transmise héréditairement sur un mode récessif autosomal. Une trentaine d'affections de ce genre sont actuellement connues. Elles sont rares, leur fréquence dans une population mixte normale n'étant que de quelques cas pour 10000 naissances. Ces enzymopathies congénitales, qui 


\section{RÉFÉRENCES}

27. Wakeed A, Pohlman R, Hasilik A, von Figura K, van Elsen A, Leroy J. Deficiency of UDP-N-acetylglucosamine : lysosomal enzyme $\mathrm{N}$-acetylglucosamine-1-phosphotransferase in organs of I-cell patients. Biochem Biophys Res Commun 1982 ; 105 : 1052-8.

28. Owada M, Neufeld EF. Is there a mechanism for introducing acid hydrolases into liver lysosomes that is independant of mannose-6phosphate recognition? Evidence from I-cell disease. Biochem Biophys Res Commun 1982 ; 105 : 814-20.

29. Wakeed A, van Etten RL. Biosynthesis and processing of lysosomal acid phosphatase in cultured human cells. Aich Biochem Biophys $1985 ; 243$ : 274-83.

30. Aerts JMFG, Brul S, Donker-Koopman WE, et al. Efficient routing of glucocerebrosidase to lysosomes requires complex oligosaccharides chain formation. Biochem Biophys Res Commun 1986 ; 141 : 452-8.

31. von Figura K, Hasilik A. Genesis of lysosomal enzyme déficiences. Trend: Biochem Sci $1984 ; 9$ : 29-31.

\section{TIRÉS A PART}

P. Crine : département de biochimie et groupe de recherche en transport membranaire, université de Montréal. portent le plus souvent sur le métabolisme des glucides, des mucopolysaccharides ou des lipides, peuvent avoir plusieurs causes. Certains syndromes sont caractérisés par une absence totale d'une hydrolase ou une forte diminution de l'activité enzymatique. Ces déficits sont généralement dus à des défauts dans la transcription du gène, à des mutations au niveau de ces gènes ou à une vitesse anormalement élevée de dégradation des hydrolases acides [31]. L'élucidation du mécanisme d'insertion des hydrolases acides dans le lysosome a permis de définir une nouvelle anomalie moléculaire au niveau de la biosynthèse du marqueur man-6-P dans le cas des mucolipidoses II. Bien que, à l'exception de greffes d'organes dans certains cas, aucun traitement efficace de ces maladies n'ait été trouvé jusqu'à présent, les progrès récents dans le domaine du transport des enzymes lysosomales renouvellent l'espoir d'un possible traitement palliatif par des préparations enzymatiques correctement " ciblées " vers les lysosomes

\section{Summary}

The molecular basis for the targeting of acid hydrolases to lysosomes has been studied extensively in the past ten years. It is now known that the oligosaccharides of newly synthesized lysosomal enzymes are phosphorylated to generate a common recognition marker : mannose-6-phosphate. This takes place after the enzyme has been transported from the endoplasmic reticulum to the Golgi apparatus. The phosphorylated acid hydrolases then bind to receptors that specifically recognize mannose-6-phosphate moieties and the enzymes bound to their receptors are transported to the lysosomes by a route that is still largely unknown. This mechanism provides a nice model which illustrates the concept that the address (or zip code) for targeting a protein to its appropriate cellular compartment is encoded in specific structural features of the protein itself. 